\title{
The utility of maraviroc, an antiretroviral agent used to treat HIV, as treatment for opioid abuse? Data from MRI and behavioural testing in rats
}

\author{
Sade C. Iriah, MPH; Catarina Borges, MSc; Uri Shalev, PhD; Xuezhu Cai, PhD; \\ Dan Madularu, PhD; Praveen P. Kulkarni, PhD; Craig F. Ferris, PhD
}

\begin{abstract}
Background: Maraviroc is an antiretroviral agent and C-C chemokine coreceptor 5 (CCR5) antagonist that is currently used to treat human immunodeficiency virus. CCR5/ $\mu$-opioid receptor heterodimerization suggests that maraviroc could be a treatment for oxycodone abuse. We treated rats with maraviroc to explore its effect on oxycodone-seeking and its interference with the analgesic effects of oxycodone. We used resting-state blood-oxygen-level-dependent functional connectivity to assess the effect of maraviroc on oxycodoneenhanced coupling in the reward circuitry and performed behavioural tests to evaluate the effect of maraviroc on oxycodone rewarding properties and on oxycodone-seeking after prolonged abstinence. Methods: Two groups of rats were exposed to 8 consecutive days of oxycodone-conditioned place preference training and treatment with maraviroc or vehicle. Two additional groups were trained to self-administer oxycodone for 10 days and then tested for drug seeking after 14 days of abstinence with or without daily maraviroc treatment. We tested the effects of maraviroc on oxycodone analgesia using a tail-flick assay. We analyzed resting-state functional connectivity data using a rat 3-dimensional MRI atlas of 171 brain areas. Results: Maraviroc significantly decreased conditioned place preference and attenuated oxycodone-seeking behaviour after prolonged abstinence. The analgesic effect of oxycodone was maintained after maraviroc treatment. Oxycodone increased functional coupling with the accumbens, ventral pallidum and olfactory tubercles, but this was reduced with maraviroc treatment. Limitations: All experiments were performed in male rats only. Conclusion: Maraviroc treatment attenuated oxycodone-seeking in abstinent rats and reduced functional coupling in the reward circuitry. The analgesic effects of oxycodone were not affected by maraviroc.
\end{abstract}

\section{Introduction}

The United States is experiencing an opioid epidemic; in 2015, about 97.5 million Americans used pain relievers, and 2 million people had a pain reliever use disorder. ${ }^{1}$ At the same time, a dramatic increase in opioid-related deaths has occurred: the number of fatalities because of drug overdose exceeded 190 cases per day in $2017 .{ }^{2}$ Clearly, there is a dire need for an effective approach that will limit the potential for abuse of opioid prescription drugs and help with the treatment of pain reliever use disorder.

A growing body of literature shows that repeated exposure to drugs of abuse results in a proinflammatory neuroimmune response that alters glia-neuron interactions. ${ }^{3}$ For example, opioids interact with $\mathrm{C}-\mathrm{C}$ chemokine co-receptor type 5 (CCR5), a G-protein-coupled receptor that is involved in immune cell function. ${ }^{4}$ Interestingly, $\mu$-opioid receptors, which mediate the effects of opioid drugs (including opioid pain relievers $),{ }^{5}$ can heterodimerize with CCR $5,{ }^{6}$ leading to an increase of CCR5 activity in the presence of opioids. This may explain why morphine given to patients infected with human immunodeficiency virus (HIV) exacerbates the infection. ${ }^{7,8}$ Moreover, acute withdrawal from morphine greatly increases the expression of endogenous CCR5 chemokine ligands, and manipulation of CCR5 alters the antinociceptive and rewarding properties of morphine. ${ }^{9}$

The mesolimbic dopaminergic system is critically involved in the rewarding and conditioned effects of opioid (and other) drugs, as well as in the development of drug abuse and addiction. ${ }^{10}$ Interestingly, recent studies have shown that Ccr5-knockout mice exhibit fewer dopamine neurons and lower levels of striatal dopamine. ${ }^{11}$ Together, the reported dysregulation of the CCR5 system by morphine and the interaction of the CCR5 system with the mesolimbic

Correspondence to: S. Iriah, Northeastern University, Center for Translational Imaging, 360 Huntington Ave., Boston, MA 02115; s.iriah@ northeastern.edu

Submitted Oct. 8, 2020; Revised Mar. 4, 2021; Revised May 31, 2021; Accepted Jul. 2, 2021

Cite as: J Psychiatry Neurosci 2021 October 8;46(5). doi: 10.1503/jpn.200191 
dopaminergic system suggest that the CCR5 system could be targeted to alter behaviours related to opioid drugs. The potential involvement of the CCR5 system in behaviours that are related to the abuse potential of drugs is supported by a recent study from Nayak and colleagues. ${ }^{12}$ The authors reported that pretreatment with maraviroc inhibited the development of cocaine-conditioned place preference (CPP) and showed evidence of CCR5 dysregulation in the nucleus accumbens and ventral tegmental area. ${ }^{12}$ Maraviroc is an antiretroviral agent and a CCR5 receptor antagonist that is currently used to treat HIV infection ${ }^{13}$ and reduce glial proinflammatory activation. ${ }^{14,15}$ Notably, maraviroc treatment does not interfere with the analgesic effects of opioid drugs; in fact, it seems to augment the analgesic properties of morphine in rats. ${ }^{16}$

In the present study, we focused on one of the most widely prescribed opioid drugs: oxycodone. Oxycodone is an easily absorbed preferential $\mu$-opioid receptor agonist $\mathrm{t}^{17}$ and a potent analgesic that was initially prescribed for the management of pain associated with surgery and palliative care, but this rapidly led to the escalation of oxycodone use and, not unexpectedly, its abuse. ${ }^{18}$ We addressed 3 issues. First, we investigated whether treatment with maraviroc would interfere with the rewarding properties of oxycodone and assessed the effects of acute maraviroc treatment on oxycodone-induced analgesia. Second, we determined whether repeated administration of maraviroc over a period of abstinence after oxycodone self-administration would attenuate oxycodone-seeking in a model of cue-induced relapse. ${ }^{19}$ Drug-seeking tests after self-administration procedures are considered the gold standard for models of drug abuse and addiction. ${ }^{20}$ Finally, repeated exposure to drugs of abuse triggers a proinflammatory neuroimmune response that is thought to at least partially mediate addiction-related brain adaptations. ${ }^{3,21} \mathrm{Ad}-$ aptations in the mesolimbic dopaminergic reward system are central to the development and maintenance of substance use disorders. ${ }^{10}$ Therefore, we sought to identify oxycodoneinduced adaptations in reward circuitry and explore the effect of pretreatment with maraviroc on these changes. Functional MRI - and more specifically, functional connectivity - is a noninvasive approach to studying the global integrated neural circuitry involved in acute and chronic drug use and the motivation behind drug-seeking behaviour and cue-induced reinstatement after withdrawal. ${ }^{22,23}$ We used resting-state blood-oxygen-level-dependent (BOLD) functional connectivity to follow changes in functional coupling in reward- and motivation-related circuitry in rats that were tested for oxycodone CPP and treated with maraviroc.

\section{Methods}

\section{Animals}

Studies were done in 2 different locations. Studies conducted at Northeastern University for CPP, tail-flick tests and functional MRI used male Sprague-Dawley rats $(n=37)$ that were purchased from Charles River Laboratories, weighed 300 to $350 \mathrm{~g}$ and were maintained on a $12 \mathrm{~h}$ light-dark cycle (lights on at 0700 h). Food and water was provided ad libitum. Rats were cared for and monitored in accordance with the guidelines published in the Guide for the Care and Use of Laboratory Animals (National Institutes of Health Publications No. 85-23, Revised 1985) and with the National Institutes of Health and American Association for Laboratory Animal Science guidelines. The protocols used in this set of experiments were compliant with the regulations of the Institutional Animal Care and Use Committee at Northeastern University.

Studies conducted at Concordia University for selfadministration and locomotor behaviour used Long Evans male rats $(n=20)$ purchased from Charles River Laboratories. Animals were housed in pairs in standard cages under a reverse $12 \mathrm{~h}$ light-dark cycle (lights off at $0930 \mathrm{~h}$ ) with food (Agribran Purina Canada Inc.) and water ad libitum and minimal environmental enrichment. All experiments were approved by the Animal Research Ethics Committee of Concordia University and were carried out in accordance with the recommendations of the Canadian Council on Animal Care.

\section{Drugs}

Oxycodone was purchased from Sigma Chemical or Toronto Research Chemicals, or it was provided as a gift by the US National Institute for Drug Abuse. It was diluted in sterile saline to achieve a dose of $3 \mathrm{mg} / \mathrm{kg}$ for i.p. injections at a volume of approximately $0.5 \mathrm{~mL}$ for CPP and the tail-flick test, and $0.1 \mathrm{mg} / \mathrm{kg}$ per infusion for self-administration.

During CPP and the tail-flick test, maraviroc (Glentham Life Science) was dissolved in sterile saline for i.p. injections of $10 \mathrm{mg} / \mathrm{kg}$. For the oxycodone-seeking test, maraviroc was dissolved in $8 \%$ dimethyl sulfoxide, $30 \%$ polyethylene glycol 400, 30\% Tween 80 and $\mathrm{H}_{2} \mathrm{O}$. The vehicle was $8 \%$ dimethyl sulfoxide, $30 \%$ polyethylene glycol $400,5 \%$ Tween 80 and $\mathrm{H}_{2} \mathrm{O}$. The drug doses we used were based on relevant literature (oxycodone ${ }^{24}$ ) and preliminary experiments in our laboratories.

\section{Behavioural procedures}

\section{CPP}

We ran a biased CPP protocol in an $80 \times 32 \mathrm{~cm}$ box, with 2 chambers characterized by distinctly different black and white patterns (checkered and diagonal stripes). The chambers were separated by a door that could be raised to permit movement between the 2 sides. A camera was placed approximately $0.6 \mathrm{~m}$ above the box, and we analyzed the recorded videos using the behavioural software ANY-maze (Stoelting Co.).

For preconditioning, on the first day animals were placed in the conditioning box with access to all compartments for $15 \mathrm{~min}$. We recorded the time spent in each compartment and analyzed it to determine which side each rat naturally preferred; this was termed the "preferred side."

Place conditioning consisted of an $8 \mathrm{~d}$ training schedule. Rats were divided into 2 groups: vehicle $(n=9)$ and maraviroc $(n=9)$. Rats received a daily dose of maraviroc $(10 \mathrm{mg} / \mathrm{kg})$ or vehicle $1 \mathrm{~h}$ before they received either oxycodone 
(3 $\mathrm{mg} / \mathrm{kg}$ ) or saline (all injections i.p.) on alternating days. Then they were placed in the conditioning chamber for $20 \mathrm{~min}$, within the oxycodone half-life. ${ }^{25}$ During these sessions, we restricted the rats to 1 compartment by closing the removable partition. On days when rats were given oxycodone, they were placed on the side they least preferred; on days when they were given saline, they were placed on their preferred side.

On the 9th day, CPP testing took place. Rats were placed in the preference box with the removable partition raised, giving them free access to both sides for $15 \mathrm{~min}$, and they were videotaped.

We analyzed the CPP data using software to avoid testing bias. We used ANY-maze software to track the time each rat spent on each side of the conditioning box, giving us an index of preference for the drug-paired side. We performed a repeated-measures analysis of variance (ANOVA) on the difference between time spent in the oxycodone-paired chamber and the saline-paired chamber (preference score), with treatment (maraviroc or vehicle) as a between-subject factor and time (pre-CPP or post-CPP) as a within-subject factor. We applied Bonferroni corrections for post hoc tests. In this report, effect sizes are presented as $\eta_{\mathrm{p}}^{2}$ or Cohen $d$.

\section{Cue-induced drug-seeking test and locomotor test}

Twenty rats were trained to self-administer oxycodone for $10 \mathrm{~d}$, in $6 \mathrm{~h}$ daily sessions. Rats were implanted with an intrajugular Silastic catheter (Dow Corning) under 2\% isoflurane anesthesia, as described by Sedki and colleagues. ${ }^{26}$ All rats were housed in the operant conditioning chamber during self-administration training. We used operant conditioning chambers with 2 retractable levers, a house light, white cue lights above the levers and a tone generator enclosed in sound-attenuating boxes $(29.0 \times 29.0 \times 25.5 \mathrm{~cm}$; Coulbourn Instruments). The first $3 \mathrm{~d}$ of training took place under a fixed ratio (FR1) schedule of reinforcement (i.e., each lever press was reinforced); the next $3 \mathrm{~d}$ were under FR2; and the last $4 \mathrm{~d}$ of training were under FR3. The daily session began with illumination of the house light, extension of the active lever and activation of the cue light and tone for $30 \mathrm{~s}$ or until the active lever was pressed. Completing the required ratio of responses (FR1, 2 or 3) on the active lever resulted in a $0.13 \mathrm{~mL}(0.1 \mathrm{mg} / \mathrm{kg})$ infusion of oxycodone over $5 \mathrm{~s}$ and the initiation of a $20 \mathrm{~s}$ time-out period during which the house light was turned off and the cue light and tone were turned on. Presses on the active lever during the time-out were recorded but did not result in an additional infusion. Presses on the inactive lever were recorded but had no programmed consequence.

After $10 \mathrm{~d}$ of oxycodone self-administration training, rats were transferred back to the Animal Care Facility and housed individually. Following a drug washout period of $24 \mathrm{~h}$, rats were assigned to 1 of 2 groups: maraviroc $(n=7)$ or vehicle $(n=6)$. The groups were matched by average number of infusions taken, active lever responses made and body weight over the last $5 \mathrm{~d}$ of training. During the $14 \mathrm{~d}$ abstinence phase, the 2 groups of rats were exposed to daily i.p. injections of maraviroc $(10 \mathrm{mg} / \mathrm{kg})$ or vehicle
(1 mL/kg). Body weight and food consumption were recorded daily.

On day 15 of abstinence, rats were returned to the operant conditioning chambers for a single $3 \mathrm{~h}$ oxycodone-seeking test session, in which drug-paired cues (the light and tone presented during oxycodone self-administration) were activated after a response on the active lever, as described for the self-administration phase. Fifteen minutes before the beginning of the test, rats were injected with either maraviroc or vehicle. The oxycodone-seeking test was performed under the same conditions as the self-administration training, except that the test was performed under extinction conditions (responses on the active lever did not result in an infusion of oxycodone). Responses on the active and inactive levers were both recorded.

On day 16 of abstinence, rats were injected with maraviroc $(10 \mathrm{mg} / \mathrm{kg})$ or vehicle $15 \mathrm{~min}$ before being placed in a locomotor activity monitoring chamber $(39 \times 42 \times 50 \mathrm{~cm}$; Coulbourn Instruments). We recorded total distance travelled (cm) over a period of $1 \mathrm{~h}$ using the TruScan software (Coulbourn Instruments).

We analyzed the mean number of infusions during selfadministration for the vehicle and maraviroc groups using 2-way ANOVA, with days ${ }^{1-10}$ as a within-subject factor and treatment (maraviroc or vehicle) as a between-subject factor. We analyzed active and inactive lever responses made over the self-administration training days using mixed-effect ANOVA, with treatment (maraviroc or vehicle) as a betweensubject factor (note: treatment groups were assigned after training) and lever (active or inactive) and days ${ }^{1-10}$ as withinsubject factors. We compared the total active and inactive lever responses performed by rats in the maraviroc and vehicle groups during the $3 \mathrm{~h}$ oxycodone-seeking test using repeated-measures ANOVA, with lever (active or inactive) as a within-subject factor and treatment (maraviroc or vehicle) as a between-subject factor. We analyzed active and inactive lever responses over time during the $3 \mathrm{~h}$ oxycodone-seeking test using 3-way repeated-measures ANOVA, with treatment (maraviroc or vehicle) as a between-subject factor and lever (active or inactive) and time (1, 2 or $3 \mathrm{~h}$ ) as within-subject factors. For the locomotor activity test, we performed repeatedmeasures ANOVA to compare the distance $(\mathrm{cm})$ travelled in $1 \mathrm{~h}$ by the maraviroc and vehicle groups, with treatment (maraviroc or vehicle) as a between-subject factor and time (6 bins of 10 minutes) as a within-subject factor. For all statistical analyses we performed, a critical threshold for statistical significance was set at $p<0.05$, using Prism (version 8.0.2; GraphPad). We applied a Geisser-Greenhouse correction for unequal variability of differences (sphericity) in the repeatedmeasures ANOVA models. For effect size calculations we used $\eta_{\mathrm{p}}{ }^{2}$ and Cohen $d$. We used Bonferroni corrections for post hoc tests.

\section{Oxycodone-induced analgesia (tail-flick test)}

The tail-flick test measures response to noxious stimuli using a heat stimulus. ${ }^{27}$ Drug-naive rats were tested before they received any treatment to obtain baseline tail-flick latency. Then, rats received a pretreatment of saline $(n=5)$ 
or maraviroc ( $n=5 ; 10 \mathrm{mg} / \mathrm{kg}) 1 \mathrm{~h}$ before a second tailflick test. Both groups were then given an i.p. injection of oxycodone ( $3 \mathrm{mg} / \mathrm{kg}) 15 \mathrm{~min}$ before the rats' tails were dipped in water set at $60^{\circ} \mathrm{C}$. If no response was detected after $10 \mathrm{~s}$, the rats' tails were removed to avoid any potential tissue damage.

We performed repeated-measures ANOVA on latency to the tail-flick test, with treatment (maraviroc or vehicle) as a between-subject factor and time (baseline or after oxycodone injection) as a within-subject factor. We applied Bonferroni correction for post hoc tests.

\section{Resting-state functional connectivity}

We imaged the rats from the CPP assay (oxycodone/vehicle and oxycodone/maraviroc) and their controls (maraviroc only; $n=5)$ and vehicle only $(n=4) 48$ hours after completion of the CPP assay. The maraviroc and vehicle controls followed the same $8 \mathrm{~d}$ treatment regimen used for the CPP assay, minus exposure to oxycodone and CPP testing. Imaging was conducted under light isoflurane anesthesia to minimize motion and physiologic stress during resting-state BOLD functional connectivity imaging (for a review, see Gorges and colleagues ${ }^{28}$ ). Anesthesia may reduce the magnitude of the BOLD signal, but it does not disrupt connectivity, as demonstrated across species and under different physiological conditions. ${ }^{29}$

We collected scans using a spin-echo triple-shot echo-planar imaging (EPI) sequence (imaging parameters: matrix size $96 \times 96 \times 20$ [height $\times$ width $\times$ depth], repetition time $1000 \mathrm{~ms}$, echo time $15 \mathrm{~ms}$, voxel size $0.312 \times 0.312 \times 1.2 \mathrm{~mm}$, slice thickness $1.2 \mathrm{~mm}, 200$ repetitions, acquisition time $10 \mathrm{~min}$ ). The benefits of multi-shot EPI in BOLD imaging have been described previously..$^{30-34}$ We avoided using single-shot EPI because of its severe geometrical distortion at high field strengths $(\geq 7 \mathrm{~T})$ and loss of effective spatial resolution as the readout period increases. ${ }^{31,35,36}$ Single-shot EPI also comes with the possibility of signal loss as a result of accumulated magnetic susceptibility or field inhomogeneity. ${ }^{34}$ Information on our data processing and analysis has been published previously $^{37}$ and is provided in Appendix 1, available at jpn.ca.

\section{Results}

\section{Behavioural models}

\section{CPP}

Figure 1 shows that exposure to oxycodone led to a significant increase in time spent in the oxycodone-paired chamber after conditioning (time: $F_{1,16}=80.67, p<0.001, \eta_{\mathrm{p}}{ }^{2}=0.834$ ). We found no overall significant effect of treatment $\left(F_{1,16}=2.946, p=\right.$ $\left.0.11, \eta_{\mathrm{p}}{ }^{2}=0.155\right)$. However, the rats treated with maraviroc during CPP exhibited a significant reduction in time spent in the drug-paired chamber after CPP (time $\times$ treatment: $F_{1,16}=$ $6.128, p=0.025, \eta_{\mathrm{p}}{ }^{2}=0.277$ ). Post hoc tests revealed a significant difference between rats treated with maraviroc and those treated with vehicle in terms of time spent in the oxycodonepaired chamber after CPP $(p=0.018, d=1.203)$.

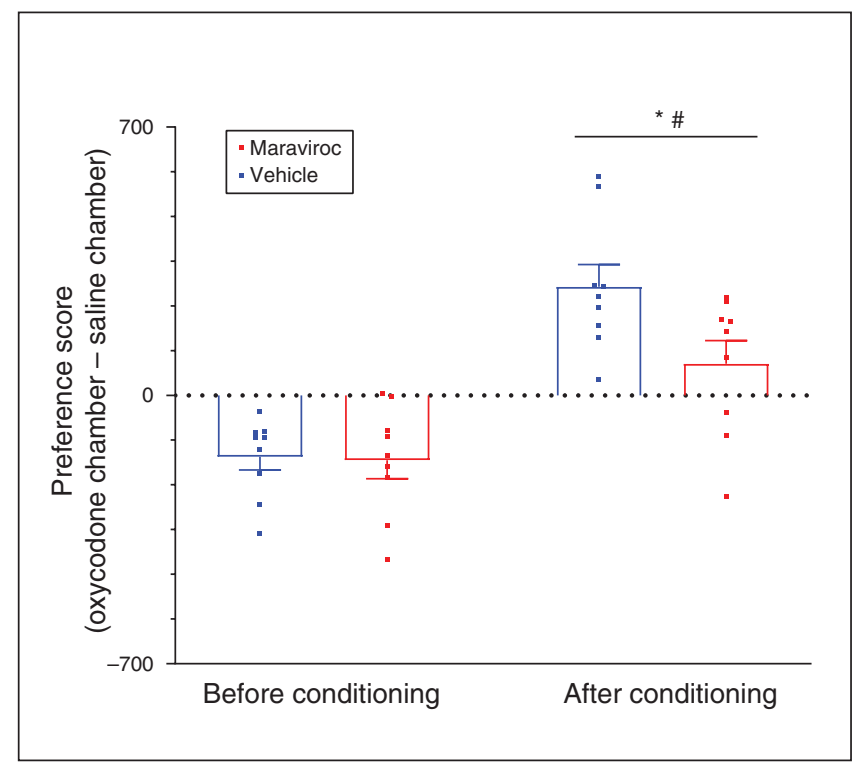

Figure 1: Conditioned place preference induced by $3 \mathrm{mg} / \mathrm{kg}$ of oxycodone. Rats were pretreated with either maraviroc or vehicle and then paired to a specific side of the chamber with oxycodone on alternating days over an $8 \mathrm{~d}$ period. \#Denotes a significantly higher preference for the side paired with oxycodone after conditioning (time: $F_{1,16}=80.67, p<0.001, \eta_{p}^{2}=0.834$ ). *Denotes a significantly lower preference for the oxycodonepaired chamber in maraviroc-treated rats compared to vehicletreated rats $(p=0.018, d=1.203)$.

\section{Cue-induced drug-seeking test}

Seven rats were excluded from analyses because they failed to reach the training criterion (an average of 10 infusions on the last $3 \mathrm{~d}$ under fixed ratio 3 , in which the ratio of active to inactive lever presses was 2 to 1$)$. All analyses were performed on a total of 13 rats (Figure 2A; maraviroc: $n=7$; vehicle: $n=6$ ). We found no significant differences between groups for mean number of oxycodone infusions, and the number of infusions remained consistent over time (days: $F_{9,99}=1.76, p=0.08, \eta_{\mathrm{p}}{ }^{2}=0.14$; treatment: $F_{1,11}=0.0001, p=$ $0.99, \eta_{\mathrm{p}}{ }^{2}=0.000$; days $\times$ treatment: $F_{9,99}=0.69, p=0.71, \eta_{\mathrm{p}}{ }^{2}=$ 0.06; Figure 2B). As shown in Figure $2 \mathrm{C}$, responses on the active lever increased from day 1 to day 10 of training, but the number of inactive lever responses remained low and stable over the $10 \mathrm{~d}$ (days: $F_{9,99}=4.11, p<0.001, \eta_{\mathrm{p}}{ }^{2}=0.272$; levers: $F_{0.283,3.111}=10.77, p=0.048, \eta_{\mathrm{p}}{ }^{2}=0.49$; days $\times$ levers: $\left.F_{2.557,25.85}=4.44, p=0.016, \eta_{\mathrm{p}}^{2}=0.305\right)$. We observed no other significant main effects or interactions.

During the oxycodone-seeking test, the number of inactive lever responses was significantly lower than the number of active lever responses (lever: $F_{1,11}=37.21, p<0.001, \eta_{\mathrm{p}}{ }^{2}=$ 0.772; Figure 3), indicating well-conditioned oxycodoneseeking. Repeated treatment with maraviroc attenuated responses on both levers compared to the vehicle-treated rats (treatment $F_{1,11}=5.72, p=0.036, \eta_{\mathrm{p}}{ }^{2}=0.342$ ). The interaction between lever and treatment was not significant (lever $\times$ treatment: $\left.F_{1,11}=2.25, p=0.162, \eta_{\mathrm{p}}{ }^{2}=0.17\right)$; however, after Bonferroni post hoc correction, the difference in active (but not inactive) lever responses between the maraviroc and 
A

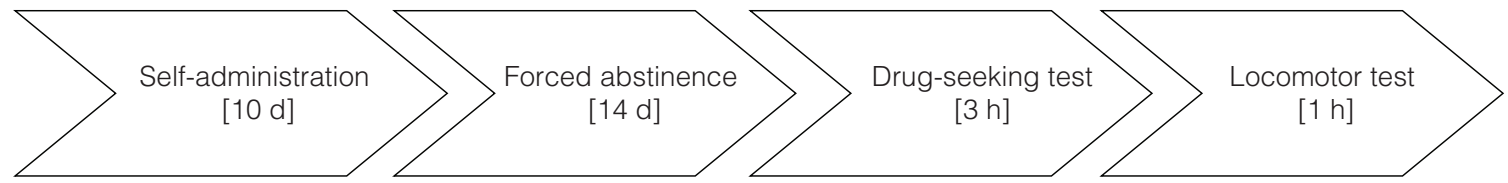

B

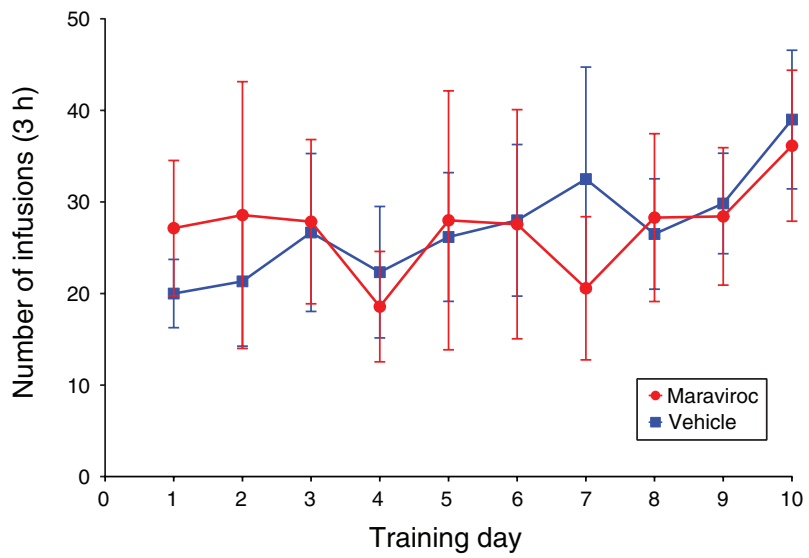

C

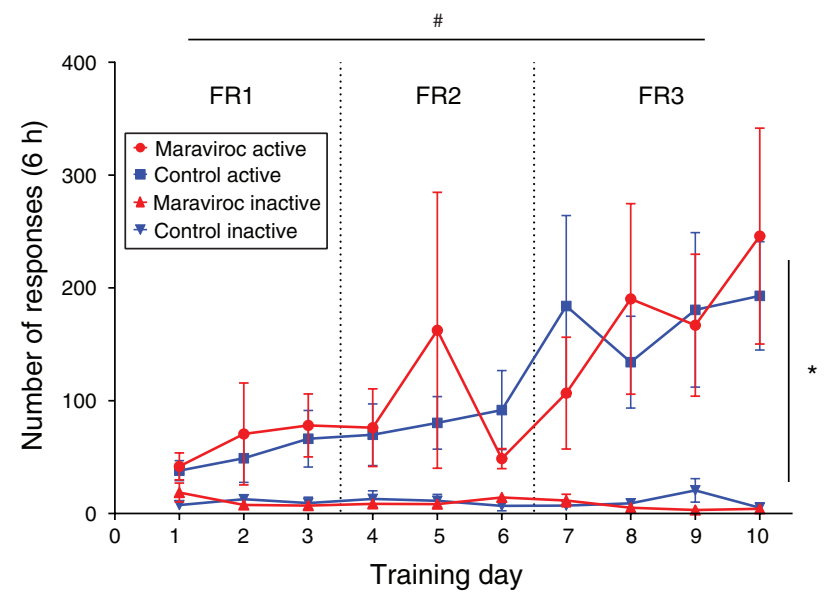

Figure 2: Oxycodone self-administration. (A) Experimental timeline for self-administration, drug-seeking tests and locomotor test. (B) Mean ( \pm standard error of the mean) number of oxycodone infusions $(0.1 \mathrm{mg} / \mathrm{kg}$ per infusion) during the $10 \mathrm{~d}$ of self-administration training for the vehicle (blue squares) and maraviroc (red circles) groups. We found no significant differences between the groups in mean number of oxycodone infusions during self-administration training. (C) Mean ( \pm standard error of the mean) responses on the active and inactive levers over $10 \mathrm{~d}$ of oxycodone self-administration $(0.1 \mathrm{mg} / \mathrm{kg}$ per infusion), under 3 fixed ratio (FR) schedules of reinforcement: FR1, FR2 and FR3 ( $n=$ 13). \#Indicates a significant increase in lever presses over training days (days: $F_{9,99}=4.11, p<0.001, \eta_{\mathrm{p}}{ }^{2}=0.272$ ). ${ }^{*}$ Denotes a significantly higher level of response on the active compared to the inactive lever (lever: $F_{0.283,3.111}=10.77, p=0.048, \eta_{\mathrm{p}}^{2}=0.49$ ).

vehicle groups was significant (active lever: $p=0.021, d=$ 1.22; inactive lever: $p=0.84, d=1.77$ ).

When we examined lever responses over the test time ( $1 \mathrm{~h}$ bins; Figure 4$)$, we found that the number of responses on both levers declined over time (time: $F_{2,22}=18.96, p<$ $0.001, \eta_{\mathrm{p}}{ }^{2}=0.633$; time $\times$ lever: $F_{1.737,19.11}=16.92, p<0.001$, $\eta_{\mathrm{p}}{ }^{2}=0.606$; time $\times$ treatment: $F_{2,22}=4.93, p=0.017, \eta_{\mathrm{p}}{ }^{2}=$ 0.31 ). The attenuation of response in maraviroc-treated rats seemed more robust for the active lever (time $x$ lever $x$ treatment: $F_{2,22}=4.46, p=0.024, \eta_{\mathrm{p}}{ }^{2}=0.288$; Figure 4A). Follow-up 2-way ANOVAs performed on active lever responses revealed a significant effect of time $\left(F_{1.639,18.03}=20.71\right.$, $\left.p<0.001, \eta_{\mathrm{p}}{ }^{2}=0.653\right)$ and a treatment $\times$ time interaction $\left(F_{2,22}=5.409, p=0.012, \eta_{\mathrm{p}}{ }^{2}=0.33\right)$. We found no significant effect of treatment $\left(F_{1,11}=4.049, p=0.07, \eta_{\mathrm{p}}{ }^{2}=0.27\right)$. Inactive lever responses over the test (1 h bins; Figure 4B) showed a significant effect of time $\left(F_{1.195,13.15}=4.654, p=0.045, \eta_{\mathrm{p}}{ }^{2}=0.3\right)$ and treatment $\left(F_{1,11}=8.577, p=0.014, \eta_{\mathrm{p}}{ }^{2}=0.44\right)$, but we found no significant treatment $\times$ time interaction $\left(F_{2,22}=\right.$ $\left.1.190, p=0.32, \eta_{\mathrm{p}}^{2}=0.098\right)$.

\section{Locomotor test}

The distance travelled by the rats over the 1 hour test decreased quickly as the rats habituated to the arena (time: $F_{3.260,35.86}=74.14, p<0.001, \eta_{\mathrm{p}}{ }^{2}=0.87$; Figure 5). Treatment with maraviroc did not change locomotor activity (treatment: $F_{1,11}=0.130, p=0.73, \eta_{\mathrm{p}}{ }^{2}=0.012$; treatment $\times$ time: $F_{5,55}=$ $\left.2.156, p=0.07, \eta_{\mathrm{p}}{ }^{2}=0.164\right)$.

\section{Oxycodone-induced analgesia (tail-flick test)}

Figure 6 shows the tail-flick test assessing pain response before and after oxycodone injection for the maraviroc and vehicle groups. We found a clear antinociceptive effect of oxycodone (time: $F_{1,8}=152.8, p<0.001, \eta_{\mathrm{p}}{ }^{2}=0.95$ ). However, we found no significant difference in pain-like response after maraviroc treatment (treatment $\times$ time: $F_{1,8}=3.19, p=0.11, \eta_{\mathrm{p}}{ }^{2}=0.285$ ). 


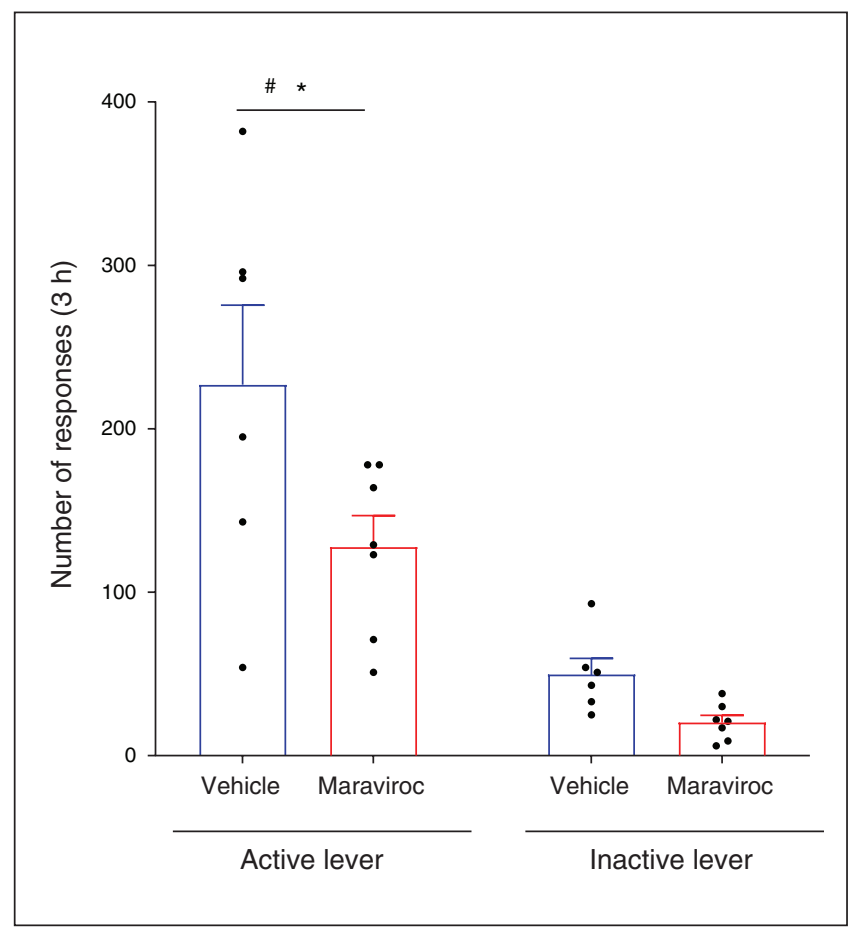

Figure 3: Difference in mean ( \pm standard error of the mean) total active and inactive lever responses for the maraviroc and vehicle groups during a $3 \mathrm{~h}$ drug-seeking test in rats trained to selfadminister oxycodone $(0.1 \mathrm{mg} / \mathrm{kg}$ per infusion) and then submitted to $14 \mathrm{~d}$ of forced abstinence. \#Denotes a statistically significant difference in active lever responses between the maraviroc and vehicle groups $(p=0.021, d=1.18)$. ${ }^{*}$ Denotes a significantly higher level of response to the active versus the inactive lever (lever: $F_{1,11}=37.21, p<0.001, \eta_{p}^{2}=0.77$ ).

\section{Resting-state functional connectivity}

Using the $k$ nearest neighbours method to identify clusters of nodes that formed resting state networks, we found that the nucleus accumbens shell had the highest number of significant connections within the cluster that reflected reward circuitry. Table 1 shows brain areas that were significantly coupled to the accumbens shell for each of the experimental conditions. In addition, all 4 experimental groups (vehicle, oxycodone, maraviroc, and maraviroc with oxycodone) showed coupling to a core of areas that included the accumbens core, the ventral pallidum and the endopiriform cortex. Vehicle-treated rats showed 11 areas that were significantly coupled to the accumbens shell, most associated with the basal ganglia, such as the medial and lateral septum and the dorsal and lateral striatum. In the oxycodone group, connectivity to the basal ganglia was lost; instead, we found an increase in connectivity to the olfactory system, the extended amygdala, the hippocampus and the dorsal prefrontal cortex that we did not observe in any of the other conditions. In the oxycodone/maraviroc group, we found loss of connection to the basal ganglia, the olfactory system, the amygdala and the hippocampus. Interestingly, maraviroc by itself still showed coupling, but it showed anticorrelation: that is, negative coupling to the ventral pallidum, the flocculus cerebellum, the locus coeruleus, the periaqueductal grey and the medial geniculate.

Data matrices comparing the different experimental conditions are provided in Appendix 1, Figure S1.

\section{Discussion}

In the experiments presented here, we used a combination of established behavioural models and brain imaging to investigate the potential of the CCR5 antagonist maraviroc as a treatment for oxycodone abuse disorder.

The first major finding in this study was that treatment with maraviroc attenuated the development of CPP, an effect that was in line with a recent report showing that cocaine CPP was significantly attenuated after treatment with maraviroc during conditioning. ${ }^{12} \mathrm{CPP}$ is thought to be a unique measure of drug reward, reflecting an approach to drugrelated environmental stimuli that can set the occasion for drug-taking behaviour. ${ }^{38}$ Importantly, a common theme in all influential theories on the development of drug addiction is the major role of the rewarding properties of drugs of abuse, leading to adaptations in the reward circuitry - primarily in the mesocorticolimbic dopaminergic pathway. ${ }^{39-41}$ Thus, CCR5 antagonism with maraviroc has the potential to interfere with drug reward and reduce abuse potential. The mechanisms through which maraviroc affects oxycodone-conditioned rewards are not known but could be related to interaction between the CCR5 and dopaminergic systems. ${ }^{42}$ An effect on the dopaminergic system is also suggested by the maravirocinduced attenuation of the CPP of cocaine, ${ }^{12}$ a psychostimulant with a direct effect on dopamine transmission. ${ }^{43}$ In contrast to the current results, maraviroc treatment did not change morphine CPP in mice. ${ }^{9}$ The reasons for this discrepancy are not clear. There were numerous differences between the 2 studies, including species (mice versus rats), the target opioid drug (morphine versus oxycodone) and the route of administration (p.o. versus i.p.), but we could not identify a specific factor that would explain the different outcomes.

Although attenuating the direct reward properties of oxycodone could help in reducing the risk for abuse, a major obstacle in the treatment of opioid addiction is the high rate of relapse, even after prolonged abstinence. ${ }^{44,45}$ Here, we used the "abstinence procedure"19 to assess the effects of maraviroc treatment on drug-seeking over a $14 \mathrm{~d}$ abstinence period in rats that had been trained to self-administer oxycodone. As previously demonstrated with psychostimulant and opiate drugs ${ }_{1}^{19}$ we observed robust oxycodone-seeking after the abstinence period. The second major finding in this study was that daily administration of maraviroc over the abstinence period substantially reduced the number of lever presses during the oxycodone-seeking test, suggesting a reduced level of oxycodone craving in the maraviroc-treated group. ${ }^{46}$ To the best of our knowledge, this was the first demonstration of the efficacy of a treatment that targets the CCR5 system on drugseeking using an operant conditioning procedure.

Opioid drug-seeking following abstinence is mediated by dopamine transmission in the mesolimbic and nigrostriatal 
A

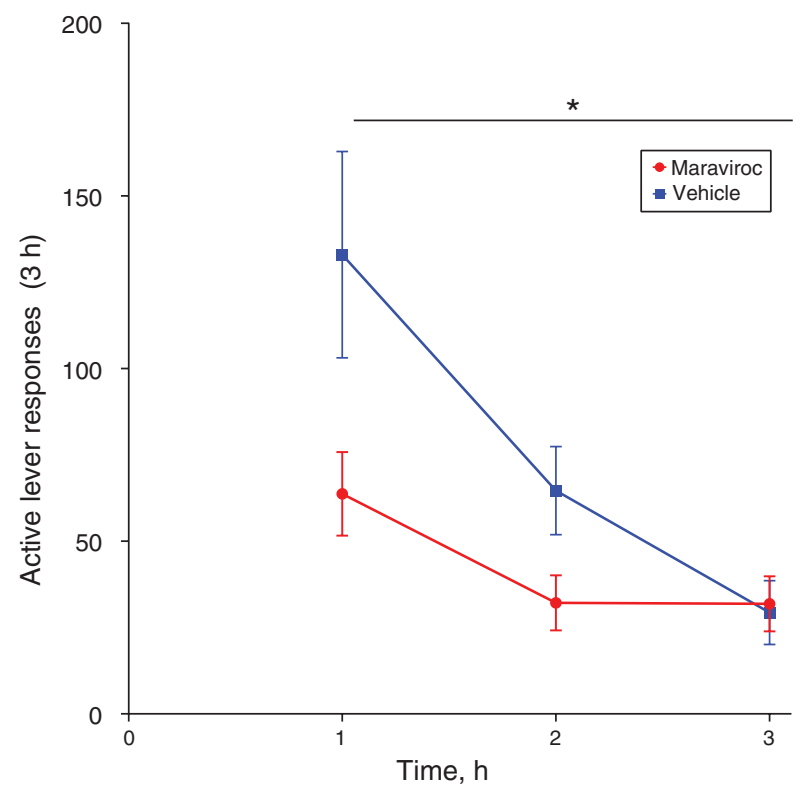

B

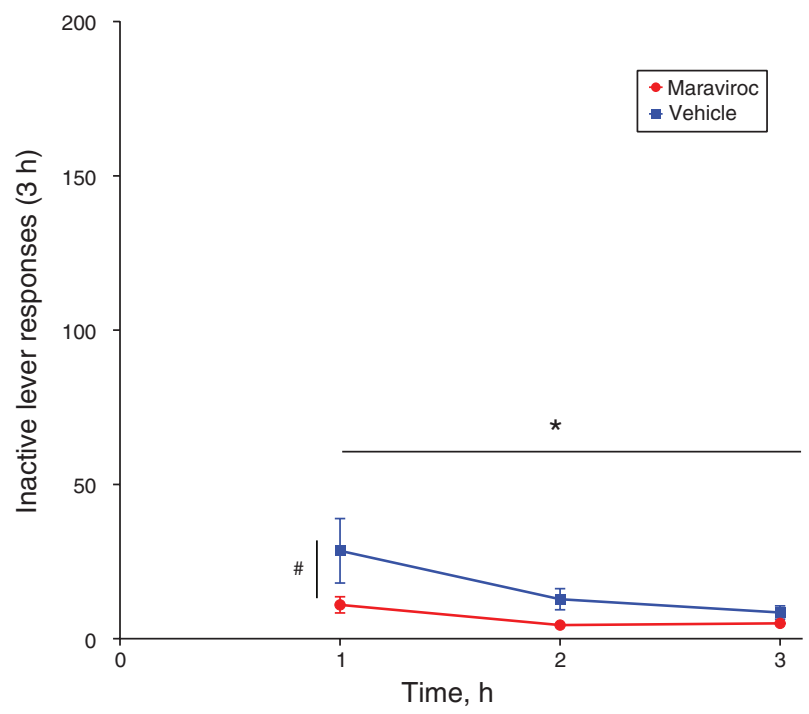

Figure 4: Oxycodone-seeking test over time. (A) Mean ( \pm standard error of the mean) active lever presses made by the vehicle $(n=6)$ and maraviroc $(n=7)$ groups during hours 1,2 and 3 of the drug-seeking test. *Denotes a significant decrease in active lever presses over time (time: $\left.F_{1.639,18.03}=20.71, p<0.001, \eta_{\mathrm{p}}{ }^{2}=0.65\right)$. (B) Mean $( \pm$ standard error of the mean) inactive lever presses made by the vehicle $(n=6)$ and maraviroc $(n=7)$ groups during hours 1,2 and 3 of the drug-seeking test. \#Represents a significant effect of treatment $\left(F_{1,11}=8.577, p=\right.$ $\left.0.014, \eta_{p}{ }^{2}=0.44\right)$. ${ }^{*}$ Denotes a significant decrease in inactive lever presses over time (time: $\left.F_{1.195,13.15}=4.654, p=0.045, \eta_{p}{ }^{2}=0.297\right)$.

pathways. ${ }^{47}$ It is therefore possible that maraviroc treatment led to interference in dopamine transmission through the CCR5 system and in turn reduced drug-seeking. ${ }^{42}$ Interestingly, we found previously that acute treatment with maraviroc had no effect on cue-induced reinstatement of oxycodone-seeking (Appendix 1, Figure S2). Direct comparisons between the 2 studies should be made with caution because of the different procedures used (reinstatement of extinguished oxycodone-seeking versus forced abstinence). However, the findings suggest that repeated exposure to maraviroc is necessary to induce the neural changes (or reverse existing adaptations) involved in its effect on oxycodone-seeking.

We also observed a significant attenuation in the number of responses on the inactive lever. The interpretation of changes in response level on the inactive lever is not always straightforward (see discussion in Shalev and colleagues ${ }^{48}$ ). Our experimental design did not allow us to completely rule out an effect of maraviroc on the expression of sensory-motor habit that developed during self-administration training. However, it is more likely that responses on the inactive lever reflect response generalization that is often observed during extinction (the condition of the oxycodone-seeking test). Other evidence of generalization of drug-seeking was the observation that although the overall number of responses on the active lever was considerably higher, we did

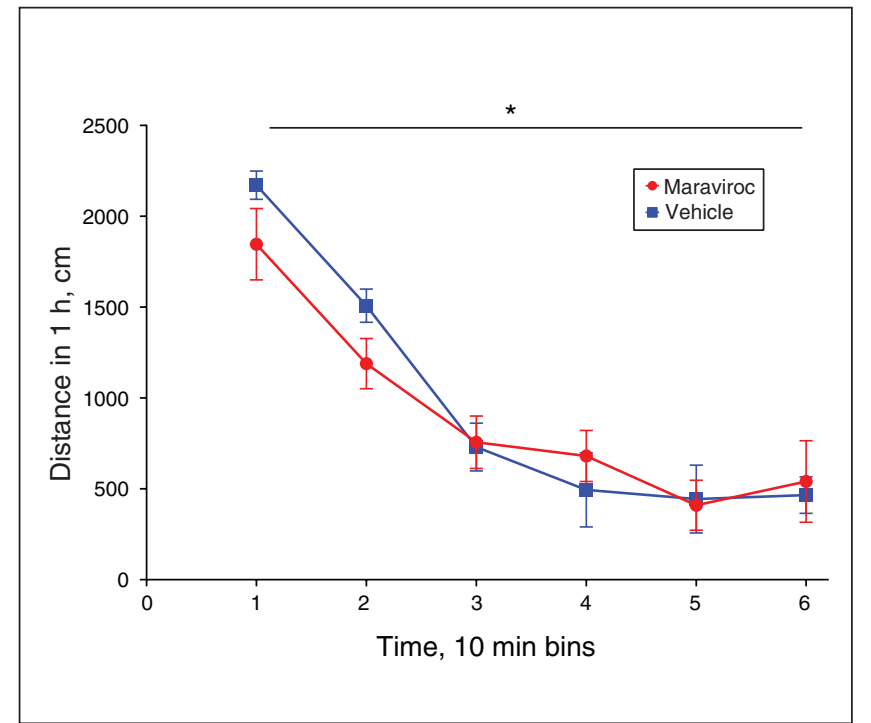

Figure 5: Locomotor activity test. Mean ( \pm standard error of the mean) distance travelled over $1 \mathrm{~h}$ (in $10 \mathrm{~min}$ bins) by the vehicle $(n=6)$ and maraviroc $(n=7)$ groups. Rats were administered maraviroc or vehicle 15 min before being placed in the activity monitoring system. We found no significant differences between groups. *Denotes a decrease in locomotor activity over time (time: $F_{3.260,35.86}=$ $\left.74.14, p<0.001, \eta_{p}^{2}=0.87\right)$. 


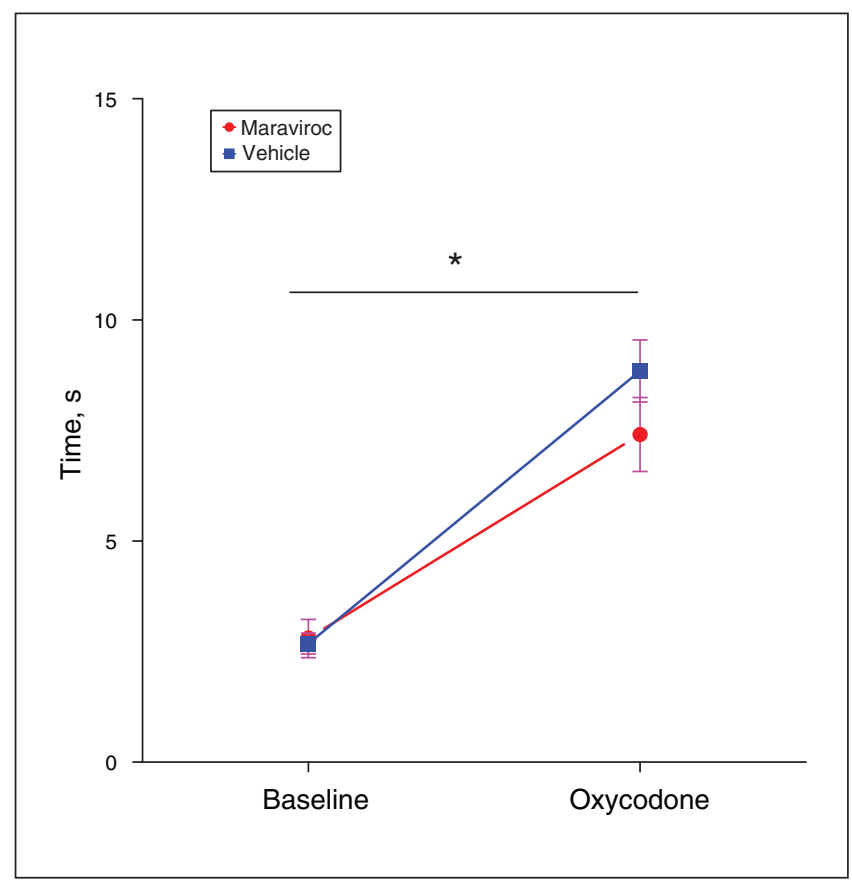

Figure 6: Oxycodone-induced analgesia. Tail-flick latencies before and after oxycodone administration ( $3 \mathrm{mg} / \mathrm{kg}$ ). The first test (baseline) showed tail-flick latencies before any drug was given in both the vehicle and maraviroc groups, and the second test (oxycodone) showed tail-flick latencies after both groups received oxycodone. Maraviroc (10 mg/kg, red) or vehicle (blue) were administered $1 \mathrm{~h}$ before the second test. *Denotes a significant increase in tail-flick latency after oxycodone treatment compared to baseline (time: $F_{1,8}=152.8, p<0.001, \eta_{p}^{2}=0.95$ ).

see an extinction pattern on the inactive lever over time. Thus, responses on both levers started to decline after the first hour once the rat realized the drug was not present. It is therefore not surprising that maraviroc treatment reduced responses on the inactive lever as well. Notably, a nonspecific motor effect was unlikely because we found no effect for maraviroc treatment on locomotor activity.

Our third major finding was that oxycodone-induced CPP was accompanied by enhanced functional coupling to key nodes in the reward circuitry (for example, the accumbens and ventral pallidum, as well as the limbic cortex, amygdala and hippocampus) in rats imaged within $48 \mathrm{~h}$ after the completion of CPP testing. This result was not unexpected and supports a neuroadaptive mechanism involving the neural circuitry of emotion and cognition that increases risk for drug-seeking behaviour after exposure to opioids. ${ }^{10}$ Rats exposed to vehicle during CPP testing showed coupling to the accumbens and ventral pallidum but not to brain areas associated with emotion and cognition. Rats exposed to oxycodone with maraviroc treatment during CPP testing showed a loss of connectivity to the basal ganglia, amygdala and hippocampus. Although our findings were correlative, they raise the possibility that the observed decoupling in the neurocircuits involved in emotion and cognition was related to the reduction in drug-seeking in maraviroc-treated rats.
To date only 2 functional MRI studies in humans have looked at the acute effect of oxycodone on functional connectivity, and both reported a decrease in functional coupling between limbic structures. ${ }^{49,50}$ We have investigated the acute effects of oxycodone on brain activity in drug-naive mice and rats and reported significant BOLD signal changes in pain neural circuitry and brain areas high in $\mu$-opioid receptors. ${ }^{51,52}$ In neither species was the accumbens responsive to this first exposure to oxycodone. It was only when rats were exposed to daily injections of oxycodone for 4 consecutive days that they showed neuronal activation in brain areas associated with drug-seeking behaviour (for example, the accumbens, the ventral striatum, the amygdala, the ventral tegmental area and the hippocampus). ${ }^{52}$ In contrast, Nasseef and colleagues examined changes in resting-state functional connectivity in rats exposed to oxycodone and reported a decrease in coupling in the periaqueductal grey and the nucleus accumbens. ${ }^{53}$ However, unlike the present report, in which rats were imaged $48 \mathrm{~h}$ after exposure to repeated oxycodone under a CPP protocol, Nasseef and colleagues applied a single exposure to oxycodone, and imaging occurred immediately after oxycodone administration. Thus, the findings reported by Nasseef and colleagues reflect acute changes in functional connectivity that involve the pain and reward centres, and not the longterm neuroadaptations that are important for the conditioned effects of drugs and drug-associated cues. Consequently, we can make 2 important observations. First, there seems to be rebound for functional connectivity in critical networks that are affected by oxycodone after a period of abstinence. Second, our data suggest that maraviroc treatment interferes with the neuroadaptive effects of repeated exposure to oxycodone on circuitry associated with emotion and cognition. Importantly, because of our experimental design we could not rule out a contribution of the exposure to the CPP procedure (i.e., the apparatus, the associative learning or both) to the observed changes in functional connectivity. Future experiments should address this caveat with appropriate control groups.

Interestingly, rats that were exposed to maraviroc alone showed loss of coupling associated with the amygdala, the limbic cortex and the hippocampus. In addition, the accumbens shell showed negative correlation or uncoupling to the ventral pallidum, the flocculus cerebellum, the locus coeruleus, the periaqueductal grey and the medial geniculate, something not seen in any of the other experimental groups. Hence, in drug-naive rats, continuous exposure to maraviroc uncoupled the basal ganglia from the accumbens shell in addition to the limbic cortex, the amygdala and parts of the hippocampus. This would suggest that maraviroc has a nonspecific effect on neurocircuitry that normally would be necessary for drug-seeking behaviour. Ccr5-knockout mice exhibit fewer dopamine neurons and lower levels of striatal dopamine. ${ }^{11}$ Thus, if repeated blockade of CCR5 results in similar adaptations, changes in coupling with these major targets of the mesolimbic dopaminergic pathway can be expected. However, we were unable to observe any nonspecific behavioural effects for maraviroc treatment. 


\begin{tabular}{|c|c|c|c|c|}
\hline Brain area & Vehicle & Oxycodone & Maraviroc & $\begin{array}{c}\text { Oxycodone/ } \\
\text { maraviroc }\end{array}$ \\
\hline Accumbens core & 3.24 & 3.48 & 2.75 & 4.23 \\
\hline Ventral pallidum & 3.31 & 4.46 & $-2.44^{*}$ & 4.37 \\
\hline Endopiriform cortex & 3.41 & 3.02 & 2.87 & 3.04 \\
\hline Claustrum & 2.58 & NS & 2.6 & 3.44 \\
\hline Medial septum & 3.36 & NS & NS & NS \\
\hline Lateral septum & 3.25 & NS & NS & NS \\
\hline Ventral orbital cortex & 2.78 & NS & NS & NS \\
\hline Dorsal medial striatum & 2.71 & NS & NS & NS \\
\hline Ventral medial striatum & 2.76 & NS & NS & NS \\
\hline Ventral lateral striatum & NS & NS & NS & 3 \\
\hline Diagonal band of Broca & 2.62 & NS & NS & NS \\
\hline Lateral orbital cortex & NS & NS & 3.08 & NS \\
\hline Insular cortex & NS & NS & NS & 3.19 \\
\hline Infralimbic cortex & 3.72 & 3.05 & 3.41 & NS \\
\hline Prelimbic cortex & NS & $2.62 \dagger$ & NS & NS \\
\hline Rostral piriform cortex & NS & 3.11 & 2.66 & NS \\
\hline Olfactory tubercles & NS & $2.66 \dagger$ & NS & NS \\
\hline Anterior olfactory nucleus & NS & $2.53 \dagger$ & NS & NS \\
\hline Intercalated amygdala & NS & $2.92 \dagger$ & NS & NS \\
\hline Central amygdala & NS & $2.83 \dagger$ & NS & NS \\
\hline Extended amygdala & NS & $2.5 \dagger$ & NS & NS \\
\hline Bed nucleus of the stria terminalis & NS & $2.86 \dagger$ & NS & NS \\
\hline Lateral preoptic area & NS & $3.91 \dagger$ & NS & NS \\
\hline Neural lobe pituitary & NS & $4.46 \dagger$ & NS & NS \\
\hline CA1 ventral hippocampus & NS & $2.55 \dagger$ & NS & NS \\
\hline Cupola cerebellum & NS & $3.96 \dagger$ & NS & NS \\
\hline Superior colliculus & NS & NS & NS & 3.03 \\
\hline Flocculus cerebellum & NS & NS & $-3^{*}$ & NS \\
\hline Locus coeruleus & NS & NS & $-3.3^{*}$ & NS \\
\hline Periaqueductal gray region & NS & NS & $-3.9^{*}$ & NS \\
\hline Medial geniculate body & NS & NS & $-2.6^{*}$ & NS \\
\hline
\end{tabular}

Although we have shown here that maraviroc can reduce CPP to oxycodone without changing oxycodone-induced analgesia, there have been reports of augmented opioid drug-induced analgesia when combined with CCR5 antagonists. It has been reported that maraviroc can reduce neuropathic pain and enhance opioid analgesia by decreasing microglia and astrocytic activation, diminishing their role in neuroinflammation-induced nociception. ${ }^{16,54}$ In an environment of neuroinflammation (a conditional Tat-expressing transgenic mouse; Tat is an HIV-1 replication regulator, but also promotes neuroinflammation), maraviroc reversed Tatinduced attenuation of morphine tolerance and symptoms of withdrawal, and it enhanced CPP to morphine. ${ }^{9}$ In addition, a ligand combining a $\mu$-opioid agonist and CCR5 antagonist greatly enhanced morphine analgesia in a mouse model of lipopolysaccharide neuroinflammation. ${ }^{55}$ These findings suggest that caution should be used when considering CCR5 antagonist-based treatment for substance use disorder in people with severe neuroinflammation.

\section{Limitations}

A major limitation of this study is that our conclusions are based on a single dose of maraviroc. The choice of a single dose of oxycodone of $3.0 \mathrm{mg} / \mathrm{kg}$ for CPP, $0.1 \mathrm{mg} / \mathrm{kg}$ per infusion for self-administration and $10 \mathrm{mg} / \mathrm{kg}$ of maraviroc was based on the literature, ${ }^{12,56,57}$ however, a thorough dose response for each of these drugs would have provided a better understanding of their interactions. In the present study, we chose to investigate the potential of maraviroc as treatment for substance use disorder using a range of behavioural and functional approaches. With the promising results reported here, future studies will use a wider range of maraviroc doses and target the mechanisms that underlie the effects of treatment with maraviroc on substance use disorder-like behaviours.

In the present study, we did not assess the development of physical dependence (withdrawal symptoms) after exposure to oxycodone in rats in the CPP or self-administration procedures. 
As well, the experiments described here involved only adult male rats. A recent report has highlighted sex differences after opioid abuse. Ryan and colleagues ${ }^{56}$ have shown sexdependent changes in opioid receptors after oxycodone$\mathrm{CPP}$, supporting the need to address sex differences in future studies. Finally, we did not perform any postmortem histology, which may have provided a better understanding of the cellular and neurochemical changes that occurred with repeated oxycodone exposure.

\section{Conclusion}

Maraviroc treatment attenuated both oxycodone-conditioned rewarding effects and oxycodone-seeking in abstinent rats. The behavioural effects we observed with oxycodone CPP and maraviroc treatment were accompanied by a reversal of increases in functional coupling in the reward circuitry in rats that were tested for oxycodone CPP. Importantly, the analgesic effects of oxycodone were not affected by this antiretroviral drug. We suggest that maraviroc could be used to reduce the risk of abuse when given as an adjunct to oxycodone to treat pain. As well, chronic treatment with maraviroc could reduce the risk of relapse in abstinent oxycodone abusers.

Affiliations: From the Centre for Translational Neuroimaging, Northeastern University, Boson, Mass., USA (Iriah, Cai, Madularu, Kulkarni, Ferris); and Concordia University, Montreal, Que., Canada (Borges, Shalev).

\section{Competing interests: None declared.}

Contributors: S. Iriah, C. Borges, U. Shalev and D. Madularu designed the study. S. Iriah, C. Borges and P. Kulkarni acquired the data, which S. Iriah, C. Borges, U. Shalev, X. Cai, P. Kulkarni and C. Ferris analyzed. S. Iriah, C. Borges, U. Shalev and C. Ferris wrote the article, which S. Iriah, C. Borges, U. Shalev, X. Cai, D. Madularu and P. Kulkarni reviewed. All authors approved the final version to be published and can certify that no other individuals not listed as authors have made substantial contributions to the paper.

Content licence: This is an Open Access article distributed in accordance with the terms of the Creative Commons Attribution (CC BY-NC-ND 4.0) licence, which permits use, distribution and reproduction in any medium, provided that the original publication is properly cited, the use is noncommercial (i.e., research or educational use), and no modifications or adaptations are made. See: https:/ / creativecommons.org/licenses/by-nc-nd/4.0/

\section{References}

1. Hughes A, Williams MR, Lipari RN, et al. Prescription drug use and misuse in the United States: results from the 2015 National Survey on Drug Use and Health. Rockville (MD): Substance Abuse and Mental Health Services Administration; 2016.

2. Hedegaard H, Minino AM, Warner M. Drug overdose deaths in the United States, 1999-2017. NCHS Data Brief 2018;329:1-8.

3. Cui C, Shurtleff D, Harris RA. Neuroimmune mechanisms of alcohol and drug addiction. Int Rev Neurobiol 2014;118:1-12.

4. Miyagi T, Chuang LF, Doi RH, et al. Morphine induces gene expression of CCR5 in human CEMx174 lymphocytes. J Biol Chem 2000;275:31305-10.

5. Bodnar RJ. Endogenous opiates and behavior: 2007. Peptides 2008; 29:2292-375.

6. Dutta R, Lunzer MM, Auger JL, et al. A bivalent compound targeting CCR5 and the mu opioid receptor treats inflammatory arthritis pain in mice without inducing pharmacologic tolerance. Arthritis Res Ther 2018;20:154.
7. Arnatt CK, Falls BA, Yuan Y, et al. Exploration of bivalent ligands targeting putative $\mathrm{mu}$ opioid receptor and chemokine receptor CCR5 dimerization. Bioorg Med Chem 2016;24:5969-87.

8. Chen C, Li J, Bot G, et al. Heterodimerization and cross-desensitization between the mu-opioid receptor and the chemokine CCR5 receptor. Eur J Pharmacol 2004;483:175-86.

9. Gonek M, McLane VD, Stevens DL, et al. CCR5 mediates HIV-1 Tat-induced neuroinflammation and influences morphine tolerance, dependence, and reward. Brain Behav Immun 2018;69:124-38.

10. Volkow ND, Morales M. The brain on drugs: from reward to addiction. Cell 2015;162:712-25.

11. Choi DY, Lee MK, Hong JT. Lack of CCR5 modifies glial phenotypes and population of the nigral dopaminergic neurons, but not MPTP-induced dopaminergic neurodegeneration. Neurobiol Dis 2013;49:159-68.

12. Nayak SU, Cicalese S, Tallarida C, et al. Chemokine CCR5 and cocaine interactions in the brain: cocaine enhances mesolimbic CCR5 mRNA levels and produces place preference and locomotor activation that are reduced by a CCR5 antagonist. Brain Behav Immun 2020;83:288-92.

13. Fadel H, Temesgen Z. Maraviroc. Drugs Today (Barc) 2007;43:749-58.

14. Lisi L, Tramutola A, De Luca A, et al. Modulatory effects of the CCR5 antagonist maraviroc on microglial pro-inflammatory activation elicited by gp120. J Neurochem 2012;120:106-14.

15. Lieberman-Blum SS, Fung HB, Bandres JC. Maraviroc: a CCR5receptor antagonist for the treatment of HIV-1 infection. Clin Ther 2008;30:1228-50.

16. Kwiatkowski K, Piotrowska A, Rojewska E, et al. Beneficial properties of maraviroc on neuropathic pain development and opioid effectiveness in rats. Prog Neuropsychopharmacol Biol Psychiatry 2016;64:68-78.

17. Lalovic B, Kharasch E, Hoffer C, et al. Pharmacokinetics and pharmacodynamics of oral oxycodone in healthy human subjects: role of circulating active metabolites. Clin Pharmacol Ther 2006;79:461-79.

18. Manchikanti L, Fellows B, Ailinani H, et al. Therapeutic use, abuse, and nonmedical use of opioids: a ten-year perspective. Pain Physician 2010;13:401-35

19. Fuchs RA, Lasseter HC, Ramirez DR, et al. Relapse to drug seeking following prolonged abstinence: the role of environmental stimuli. Drug Discov Today Dis Models 2008;5:251-8.

20. Kuhn BN, Kalivas PW, Bobadilla AC. Understanding addiction using animal models. Front Behav Neurosci 2019;13:262.

21. Lacagnina MJ, Rivera PD, Bilbo SD. Glial and neuroimmune mechanisms as critical modulators of drug use and abuse. Neuropsychopharmacology 2017;42:156-77.

22. Courtney KE, Schacht JP, Hutchison K, et al. Neural substrates of cue reactivity: association with treatment outcomes and relapse. Addict Biol 2016;21:3-22.

23. Jasinska AJ, Stein EA, Kaiser J, et al. Factors modulating neural reactivity to drug cues in addiction: a survey of human neuroimaging studies. Neurosci Biobehav Rev 2014;38:1-16.

24. Bossert JM, Hoots JK, Fredriksson I, et al. Role of mu, but not delta or kappa, opioid receptors in context-induced reinstatement of oxycodone seeking. Eur J Neurosci 2019;50:2075-85.

25. Ordonez Gallego A, Gonzalez Baron M, Espinosa Arranz E. Oxycodone: a pharmacological and clinical review. Clin Transl Oncol 2007;9:298-307.

26. Sedki F, D'Cunha T, Shalev U. A procedure to study the effect of prolonged food restriction on heroin seeking in abstinent rats. J Vis Exp 2013;81:e50751.

27. Tjølsen A, Hole K. Tail-flick test. In: Gebhart GF, Schmidt RF, editors. Encyclopedia of pain. Berlin, Heidelberg: Springer Berlin Heidelberg; 2013:3832-7.

28. Gorges M, Roselli F, Muller HP, et al. Functional connectivity mapping in the animal model: principles and applications of resting-state fMRI. Front Neurol 2017;8:200.

29. Liang Z, King J, Zhang N. Intrinsic organization of the anesthetized brain. J Neurosci 2012;32:10183-91.

30. Menon RS, Thomas CG, Gati JS. Investigation of BOLD contrast in fMRI using multi-shot EPI. NMR Biomed 1997;10:179-82.

31. Hoogenraad FG, Pouwels PJ, Hofman MB, et al. High-resolution segmented EPI in a motor task fMRI study. Magn Reson Imaging 2000;18:405-9.

32. Poser BA, Norris DG. Investigating the benefits of multi-echo EPI for fMRI at 7 T. Neuroimage 2009;45:1162-72. 
33. Swisher JD, Sexton JA, Gatenby JC, et al. Multishot versus singleshot pulse sequences in very high field fMRI: a comparison using retinotopic mapping. PLoS One 2012;7:e34626.

34. Kang D, Sung YW, Kang CK. Fast imaging technique for fMRI: consecutive multishot echo planar imaging accelerated with GRAPPA rechnique. BioMed Res Int 2015;2015:394213.

35. Farzaneh F, Riederer SJ, Pelc NJ. Analysis of $T_{2}$ limitations and offresonance effects on spatial resolution and artifacts in echo-planar imaging. Magn Reson Med 1990;14:123-39.

36. Jesmanowicz A, Bandettini PA, Hyde JS. Single-shot half k-space high-resolution gradient-recalled EPI for fMRI at 3 Tesla. Magn Reson Med 1998;40:754-62.

37. Kulkarni P, Morrison TR, Cai X, et al. Neuroradiological changes following single or repetitive mild TBI. Front Syst Neurosci 2019;13:34.

38. Bardo MT, Bevins RA. Conditioned place preference: what does it add to our preclinical understanding of drug reward? Psychopharmacology (Berl) 2000;153:31-43.

39. O'Brien CP, Gardner EL. Critical assessment of how to study addiction and its treatment: human and non-human animal models. Pharmacol Ther 2005;108:18-58.

40. Luscher C, Robbins TW, Everitt BJ. The transition to compulsion in addiction. Nat Rev Neurosci 2020;21:247-63.

41. Robinson TE, Berridge KC. The neural basis of drug craving: an incentive-sensitization theory of addiction. Brain Res Brain Res Rev 1993;18:247-91.

42. Lanfranco MF, Mocchetti I, Burns MP, et al. Glial- and neuronalspecific expression of CCL5 mRNA in the rat brain. Front Neuroanat 2017;11:137.

43. Volkow ND, Wang GJ, Fowler JS, et al. Reinforcing effects of psychostimulants in humans are associated with increases in brain dopamine and occupancy of $\mathrm{D}(2)$ receptors. J Pharmacol Exp Ther 1999;291:409-15.

44. Hser YI, Hoffman V, Grella CE, et al. A 33-year follow-up of narcotics addicts. Arch Gen Psychiatry 2001;58:503-8.

45. Gostin LO, Hodge JG Jr, Noe SA. Reframing the opioid epidemic as a national emergency. JAMA 2017;318:1539-40.

46. Reiner DJ, Fredriksson I, Lofaro OM, et al. Relapse to opioid seeking in rat models: behavior, pharmacology and circuits. Neuropsychopharmacology 2019;44:465-77.
47. Gao J, Li Y, Zhu N, et al. Roles of dopaminergic innervation of nucleus accumbens shell and dorsolateral caudate-putamen in cue-induced morphine seeking after prolonged abstinence and the underlying D1- and D2-like receptor mechanisms in rats. J Psychopharmacol 2013;27:181-91.

48. Shalev U, Grimm JW, Shaham Y. Neurobiology of relapse to heroin and cocaine seeking: a review. Pharmacol Rev 2002;54:1-42.

49. Gorka SM, Fitzgerald DA, de Wit H, et al. Opioid modulation of resting-state anterior cingulate cortex functional connectivity. J Psychopharmacol 2014;28:1115-24.

50. Hansen TM, Lelic D, Olesen AE, et al. Differential effects of oxycodone and venlafaxine on resting state functional connectivity: a randomized placebo-controlled magnetic resonance imaging study. CNS Neurosci Ther 2018;24:820-7.

51. Moore K, Madularu D, Iriah S, et al. BOLD imaging in awake wild-type and mu-opioid receptor knock-out mice reveals ontarget activation maps in response to oxycodone. Front Neurosci 2016;10:471.

52. Iriah SC, Trivedi M, Kenkel W, et al. Oxycodone exposure: a magnetic resonance imaging study in response to acute and chronic oxycodone treatment in rats. Neuroscience 2019;398:88-101.

53. Nasseef MT, Singh JP, Ehrlich AT, et al. Oxycodone-mediated activation of the mu opioid receptor reduces whole brain functional connectivity in mice. ACS Pharmacol Transl Sci 2019;2:264-74

54. Piotrowska A, Kwiatkowski K, Rojewska E, et al. Maraviroc reduces neuropathic pain through polarization of microglia and astroglia - evidence from in vivo and in vitro studies. Neuropharmacology 2016;108:207-19.

55. Akgun E, Javed MI, Lunzer MM, et al. Inhibition of inflammatory and neuropathic pain by targeting a mu opioid receptor/ chemokine receptor5 heteromer (MOR-CCR5). J Med Chem 2015; 58:8647-57.

56. Ryan JD, Zhou Y, Contoreggi NH, et al. Sex differences in the rat hippocampal opioid system after oxycodone conditioned place preference. Neuroscience 2018;393:236-57.

57. Blackwood CA, Leary M, Salisbury A, et al. Escalated oxycodone self-administration causes differential striatal mRNA expression of FGFs and IEGs following abstinence-associated incubation of oxycodone craving. Neuroscience 2019;415:173-83. 